such control are firmly established and widely known. Its application is proving increasingly successful though it is necessary to adjust the principles to meet the varying demands of the various industries.

A second group of papers deals with scientific methods applied to works management in a number of specific examples.

Distribution is becoming very definitely a science. It is axiomatic that the manufacturer of a new product must sell it and that the true market is always the ultimate user. A paper contributed by the Advertising Association of Great Britain gives a particularly good summary of the routes through which a new product can be distributed to the public, with concrete examples of recent introductions. Any reader of this volume who has the task of introducing a new product can easily ascertain the international views as to how he should begin his task. Large organisations are finding it worth while to establish special departments for marketing research : some of the methods employed and results obtained in the United States are described by Mr. O. F. Roost. As one industry after another reaches a point where it is able to produce more than the market can apparently consume, competition becomes keener and the need for more intimate and accurate knowledge of existing markets and possible new ones becomes apparent.
E.F.A.

\title{
The Metallic State
}

A CONFERENCE on "The Metallic State" was held in the University of Bristol at the H. H. Wills Physics Laboratory on July 2-5, about forty visitors being present from other parts of England and from the Continent. Most of the visitors were housed in Wills Hall, one of the University halls of residence.

At the opening session of the Conference, Dr. J. A. Prins (Groningen) gave an account of the results obtained at the University of Groningen on the absorption and emission of X-rays by metals. The absorption spectrum in crystalline solids has a fine structure extending several hundred electron volts on the short wave-length side of the edge, and depending in its general features only on the structure, and not on the atomic constitution of the crystal. A theoretical explanation due to Kronig was discussed, which relates the phenomenon to the Bragg reflection of the ejected electrons. Dr. H. W. B. Skinner (Bristol) showed how the study of emission bands in the ultrasoft region can give information about the occupied electronic states in metals. Prof. W. L. Bragg (Manchester) gave a report of some recent results obtained by Sykes on the formation of a superstructure in copper. gold alloys, and Mr. J. D. Bernal (Cambridge) discussed the factors which determine the crystal structure of alloys, pointing out especially that the atomic radii of the constituents have often a decisive influence.

The morning of July 3 was devoted to a discussion of the liquid and amorphous states. Prof. H. Mark (Vienna) gave an account of recent theories of electrical conductivity, and Dr. J. A. Prins gave a report on his own work on amorphous antimony. Dr. G. W. Brindley (Leeds) showed the effect of filing on the intensity of X-ray reflection from metals. In the afternoon there was a discussion on the properties of metallic bismuth, in which Prof. A. Goetz (Pasadena), Dr. N. Thompson (Bristol), Dr. D. Shoenberg (Cambridge) and Dr. H. Jones (Bristol) took part. Bismuth is peculiar in that very small quantities (less than 0.1 per cent) of lead, tin or other metals in solid solution have a very marked effect on its electrical conductivity and diamagnetic susceptibility. Prof. Goetz expressed the opinion that these facts suggest the existence in bismuth of a microcrystalline superstructure of the kind postulated by $Z_{w i c k y}$, but an alternative explanation was suggested in terms of the wave mechanical theories due to Pejerls and Jones. These theories, however, will require further development before any quantitative account of the facts can be given.

On July 4, Prof. W. Gerlach (Munich) gave an account of the work carried out in his laboratory on the electrical resistance of nickel and of some of its alloys. The resistance of nickel decreases in a magnetic field, the decrease being proportional to the change in $\sigma^{2}$, where $\sigma$ is the magnetisation. Further, the resistance plotted against temperature shows a kink at the Curie point. A possible explanation of the effect was suggested by Prof. N. F. Mott (Bristol). Dr. E. C. Stoner (Leeds) pointed out that various methods of determining the Weiss intramolecular field constant in nickel give very discordant results, and suggested an explanation.

On July 5, a discussion on supra-conductivity was opened by Dr. J. M. Casimir (Leyden), who gave an account of the fundamental experiments of Meissner, and of de Haas and Mrs. Casimir, and of the important theoretical advances of Gorter, Casimir and London. Mr. K. Mendelssohn (Oxford) gave an account of the behaviour of certain alloys. It appears that though great progress has been made towards the understanding of the thermodynamics and electrodynamics of a supra-conductor, we are still as far as ever from knowing the cause of the phenomenon. Dr. $H$. Niewodniczański and Mr. C. J. Milner (Mond Laboratory, Cambridge) discussed the electrical conductivity of non-supra-conductors, and here also the results of theory are by no means in accord with experiment, and the opinion was expressed on several sides that all theories are wrong below $4^{\circ} \mathrm{K}$. Prof. F. Simon (Oxford) and Dr. E. Teller (London) also discussed the possibility of using nuclear spins to obtain yet lower temperatures than can be obtained with ordinary paramagnetics by the magnetic cooling method.

An interesting feature of the Conference was the frequent mention of experimental facts for which no quantitative theoretical explanation could be given. Considerable advances have recently been made in the quantum theory of the metallic state, and it is now possible for the theorist to give an indication of why certain metals have their particular crystal structures, magnetic properties and electrical conductivities. Nevertheless, the theory is as yet far from being able to give such a complete account of the experimental data as is possible, for example, in the field of atomic spectra. 